\title{
R-bUCRP: A Novel Reputation-Based Uneven Clustering Routing Protocol for Cognitive Wireless Sensor Networks
}

\author{
Mingchuan Zhang, Ruijuan Zheng, Ying Li, Qingtao Wu, and Liang Song \\ Information Engineering College, Henan University of Science and Technology, Luoyang 471023, China \\ Correspondence should be addressed to Mingchuan Zhang; zhang_mch@haust.edu.cn and Ruijuan Zheng; rjwo@163.com
}

Received 23 March 2016; Revised 17 June 2016; Accepted 28 June 2016

Academic Editor: Iftikhar Ahmad

Copyright ( 2016 Mingchuan Zhang et al. This is an open access article distributed under the Creative Commons Attribution License, which permits unrestricted use, distribution, and reproduction in any medium, provided the original work is properly cited.

Energy of nodes is an important factor that affects the performance of Wireless Sensor Networks (WSNs), especially in the case of existing selfish nodes, which attracted many researchers' attention recently. In this paper, we present a reputation-based uneven clustering routing protocol (R-bUCRP) considering both energy saving and reputation assessment. In the cluster establishment phase, we adopt an uneven clustering mechanism which controls the competitive scope of cluster head candidates to save the energy of WSNs. In the cluster heads election phase, the residual energy and reputation value are used as the indexes to select the optimal cluster head, where the reputation mechanism is introduced to support reputation assessment. Simulation results show that the proposed R-bUCRP can save node energy consumption, balance network energy distribution, and prolong network lifetime.

\section{Introduction}

As a new ubiquitous network, Wireless Sensor Networks (WSNs) are widely used in various fields, such as ecological protection, energy conservation and emission reduction, and food security. As a huge network, WSNs have diverse sensor nodes and multifarious communication protocols. The resource management for WSNs is difficult and the application fields are extensive. Meanwhile, the complexity and uncertainty promote the idea that cognition becomes an important characteristic for WSNs.

In 1999, Dr. Mitola and Maguire Jr. [1] proposed the concept of cognitive radio and integrated "cognitive" elements into radio networks, which has attracted a lot of researches and has achieved many research results. The thought of cognition promoted the development of network intelligence greatly. Cognitive radio network [2, 3], cognitive network [46], and Cognitive Internet of Things [7, 8] have greatly interested the researchers, and large numbers of achievements have been attained, which greatly promoted the evolution of network intelligence.

In recent years, with the rapid development of the WSNs, the WSNs' intelligent feature has been involved in more and more researches. The concept of the Cognitive Wireless
Sensor Networks (CWSNS) is derived from the autonomic computing [9] and bioinspired theory [10], which puts the "cognitive" element into the WSNs. Since its core idea is to put the intelligent characteristic into IoT, CIoT has the characteristics of self-perception, self-decision, self-learning, self-optimization, and self-adjustment. For network communications, although nodes should cooperate with each other and provide services for others, the existing research shows that a large number of nodes only want to enjoy the services provided by other nodes due to the limited energy of nodes but do not want to provide services for others [11]. Since CWSNS is a large scale network with limited energy and different performance goals in different regional network, there could be many selfish nodes which would cause negative forwarding of packets. Scientific researches prove that forward selfish behavior will affect seriously the network performance-just only a small number of selfish nodes $(10 \%-40 \%)$ will lead to a significant decrease $(16 \%-$ $32 \%)$ of network performance [12]. Therefore, how to design a reliable routing mechanism is a key problem to be solved in the information transmission field of CWSNS.

Considering the characteristics of scalability and energy saving in networks, the clustering method is always applied to the heterogeneous Wireless Sensor Network protocols, 
especially in the large scale network. The clustering method provides a hierarchical structure for the network, which has two members, the cluster head nodes and the cluster member nodes. The cluster head nodes have better basic conditions and manage their cluster member. They fuse the information that cluster member nodes collected and then send it to the base station. In the early stage, the route clustering often used even clustering method. The typical even clustering protocols include LEACH [13], TEEN [14], and APTEEN [15]. Subsequently, Bhasker [16] proposes a genetically derived secure cluster-based data aggregation in WSN which minimizes the energy consumption, ensures data security, and reduces the transmission overhead. Lin and Uster [17] consider a mixed-integer linear programming (MILP) model to optimally determine the sink and $\mathrm{CH}$ locations as well as the data flow in the network, which could prolong the network lifetime. Quang and Kim [18] propose a clustering algorithm to enhance the performance of fixed wireless sensor and actuators networks (WSANs). In each cluster, a multilevel hierarchical structure can be applied to reduce the energy consumption. In addition to the cluster head, some nodes can be selected as intermediate nodes, which manages a subcluster. Li and Hua [19] propose a clustering algorithm for energy-efficiency in Wireless Sensor Networks, which is partially similar to this paper. The requirements of even clustering method are even size of clusters in networks and the same number of nodes in the cluster. Polling method used in cluster head election could balance the energy consumption. However, data collection, data fusion, and communication with the base station are usually completed alone by the cluster head node, which will cause premature death, energy consumption imbalance, and short network lifetime.

In order to solve the problem, many researchers have focused in their works on the uneven clustering method to balance the energy consumption. EECS [20] not only considers the distance between cluster member nodes and cluster head nodes, but also considers the distance of the cluster head nodes to the base station and constructs uneven size of clusters to ease the problem of the uneven energy consumption. UCS [21] balanced the energy consumption of the cluster head nodes and adjusted the size of the cluster according to the expected forwarding load of the cluster head nodes. EEUC [22] made use of the uneven size of competitive radius. The number of the cluster members near the base station is relatively small, so that the cluster head nodes could save energy for the cluster data forwarding. It optimizes the energy consumption of nodes and prolongs the network lifetime significantly. CEB-UC [23] showed the reasonable partition of the network area. The closer to the base station clusters are, the less sensor nodes in each cluster are, so that the energy consumption of nodes in the network is balanced. Jiang et al. [24] achieved the uneven clustering by uneven layering of the sensor network model. Each layer forms the cluster independently to balance the energy consumption of cluster head nodes and prolong the network lifetime. Buchegger and Le Boudec [25] used cluster head competitive algorithm based time rules. The broadcast time depends on the residual energy of the cluster head candidate nodes and their neighbor nodes. The energy consumption of the nodes could be compensated by each other.

The above researches use energy and distance to elect cluster heads and clustering. However, the reliability of the cluster head nodes is also very important. The reputation value of the nodes is often used to measure the reliability of the nodes. The higher the reputation value of the cluster head nodes is, the more reliable the nodes are and the higher the quality of service provided by the nodes in the cluster is. Naghizadeh and Liu [26] describe the reputation problem in a distributed multiuser system by using a mechanism design theoretic approach. They introduce a number of utility models representing users' strategic behavior and design a mechanism that achieves the optimal performance. Due to the node collusion impairing the effectiveness of reputation systems in trustworthy node selection, Li et al. [27] identify suspicious collusion behavior by examining the overstock transaction trace of reputation ratings and propose the Social Trust mechanism that leverages a social network to combat collusion. Refaei et al. [28] introduce a time-slotted approach to allow the evaluation function to quickly and accurately capture changes in node behavior and show the ability to achieve high accuracy and promptness in dynamic environments. Labraoui et al. [29] propose a Risk-aware Reputation-based Trust (RaRTrust) model for WSNs, using both reputation and risk to evaluate trustworthiness of a sensor node. Capossele et al. [30] propose a reputation-based channel aware routing protocol (R-CARP) for underwater acoustic sensor networks (UASNs). It was tailored to such communication constrained environment. The confident protocol proposed in [31] is a kind of ad hoc nodes reputation evaluation mechanism. Through the monitoring of the neighbor nodes, the reputation value is calculated and compared with the presetting threshold to judge the reliability of the nodes. Heinzelman et al. [13] used the watchdog mechanism to monitor the next hop node's forwarding behavior. If the next node would not transmit the data packet, it is indicated that the node's reliability is low. Moreover, the reputation values recommended by other nodes are taken into account for collaborative computing node's reputation value.

We presented a next hop selecting algorithm for static sensor nodes in [32], a "Physarum and ants" based hybrid routing algorithm for WSNs [33], and a reputation revision algorithm in cloud circumstance [34]. In this paper, we propose a reputation-based uneven cluster routing protocol (R-bUCRP) based on the prior works. The proposed protocol introduces the reputation mechanism firstly; next calculates the value of the node's direct reputation and relative reputation according to the node's historical interaction information and the similarity of the collected data; then evaluates comprehensively the reputation in consideration of the node's income and expenses value when it joins into the cluster; and finally details the cluster heads election method according to the weight value of the cluster head candidate node's residual energy and reputation value. The proposed method could efficiently decrease the dead speed of the nodes, balance the energy consumption of all nodes, and prolong the network lifetime. 


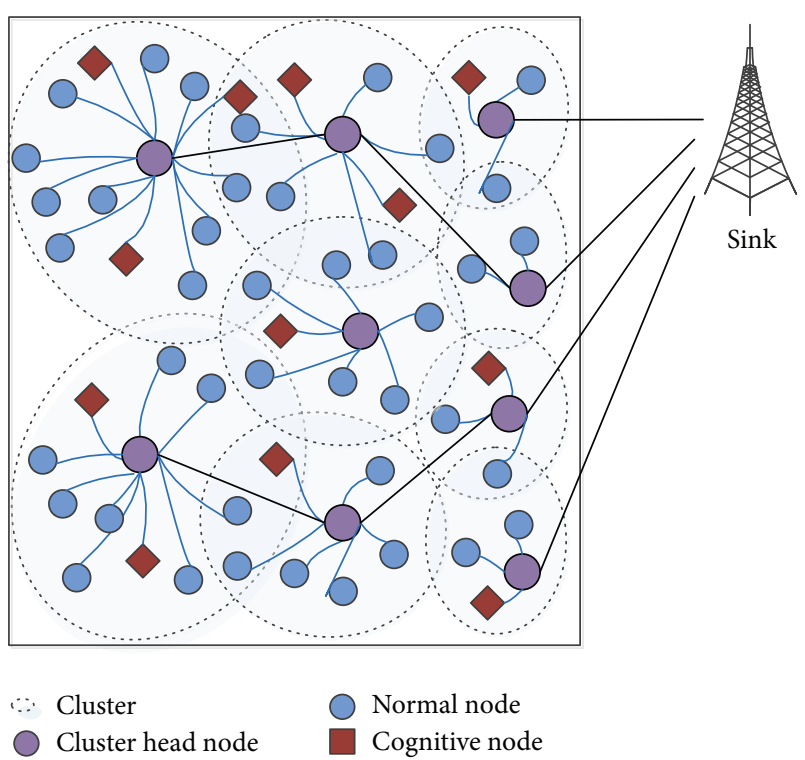

Figure 1: Network model.

The rest of the paper is organized as follows. Section 2 introduces network clustering model, energy model, and the reputation model. Section 3 details the proposed R-bUCRP. Section 4 evaluates our models and algorithms with extensive simulations. Finally, the conclusion is presented in Section 5.

\section{System Models}

2.1. CWSNS Model. In this paper, we construct a network model for CWSNS, as shown in Figure 1. The CWSNS is divided into multiple clusters based on the cluster model of Wireless Sensor Network. We use the uneven clustering method in this paper. The closer to the base station a cluster is, the smaller its radius is. Nodes in the network are divided into cluster head nodes and cluster member nodes. The cluster head is elected according to the cluster head competitive rules, such as more residual energy and less energy consumption. The cluster head node manages cluster member nodes, fuses the information collected by cluster members, and sends it to the base station. The cluster member nodes include cognitive nodes and normal nodes. Cognitive nodes are special normal nodes where "cognitive" element is introduced. Cognitive nodes have the characteristics of wisdom, perceive the change of environment, and make the corresponding decision.

Here, we present some assumptions for the network model used in the proposed method as follows.

(i) Each node has a unique ID.

(ii) The energy of the node is heterogeneous, is limited, and cannot be supplied, and the node could obtain its own current energy.

(iii) The base station cannot be moved, which has infinite energy and strong capacity of computing and storage.

(iv) The data fusion technology is used to reduce the amount of data transmission.

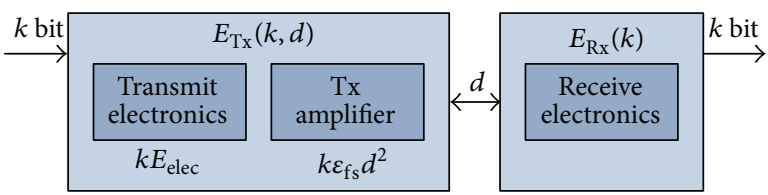

FIGURE 2: Circuit model of sending and receiving data.

(v) The node is not provided with Global Positioning System (GPS) function module, so it has no position sensing capability.

2.2. Energy Consumption Model. In this paper, the energy consumption model of wireless communication is adopted from [22], where both the free space and multipath fading channels are used depending on the distance between the transmitter and receiver node. If the communication distance is less than a threshold value $d_{0}$, the free space model is used. Otherwise, the multipath fading model is considered. The receiving and sending circuit models of the wireless sensor nodes are shown in Figure 2.

As shown in Figure 2, the energy consumption for transmitting data is mainly composed of two parts, that is, transmitting circuit and power amplifier circuit. The energy consumed by node $i$ to transmit $k$ bit data packet to node $j$ with the distance $d$ is given in

$$
E_{\mathrm{Tx}}(k, d)= \begin{cases}k E_{\text {elec }}+k \varepsilon_{\mathrm{fs}} d^{2}, & d<d_{0} \\ k E_{\text {elec }}+k \varepsilon_{\mathrm{mp}} d^{4}, & d \geq d_{0} .\end{cases}
$$

The energy consumption for receiving data comes mainly from receiving circuit, so the energy consumed by node to receive $k$ bit data packet is shown in

$$
E_{\mathrm{Rx}}(k)=k E_{\text {elec }} \text {, }
$$

where $E_{\text {elec }}$ is the radio frequency energy consumption coefficient and $\varepsilon_{\mathrm{fs}}$ and $\varepsilon_{\mathrm{mp}}$ are energy required by amplifier in free space and multipath, respectively. Through (1), $d_{0}$ is given as $d_{0}=\sqrt{\varepsilon_{\mathrm{fs}} / \varepsilon_{\mathrm{mp}}}$.

2.3. Reputation Model. Reputation mechanism provides an effective way to judge the trust relationship between nodes. The introduction of reputation can help determine the degree of cooperation between the nodes, identify the selfish nodes efficiently, and punish or incent the selfish nodes in some way. The so-called selfish nodes are the nodes which do not forward data or forward selectively data in order to maintain their own energy. Assuming that the node $i$ is the neighbor node of node $j$, if $i$ wants to know whether $j$ is reliable or not, it could determine by computing the reputation value of $j$.

The traditional node reputation calculation mainly considers two aspects: direct reputation value and indirect reputation value. The reputation evaluation process is shown in Figure 3. Direct reputation means that the reputation value of $j$ is evaluated based on the direct historical interaction between nodes $i$ and $j$, while indirect reputation value of $j$ is 


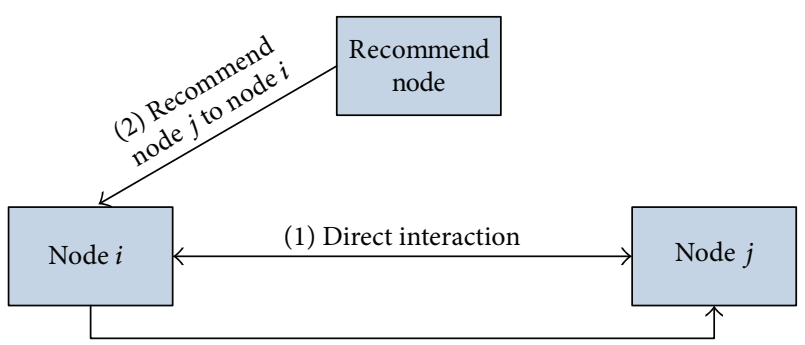

(3) Comprehensive reputation evaluation

FIgURE 3: Reputation evaluation process.

formed from the recommended value according to the other nodes' reputation to $j$.

\section{R-bUCRP Description}

In the network establishment phase, the base station needs to broadcast a signal with a given transmit power. Each sensor node calculates the approximate distance to the base station based on the intensity of the received signal. The distance is not only helpful for the nodes to select the appropriate transmit power to save energy consumption, but also as one of the necessary pieces of information to construct clusters.

The basic process of the R-bUCRP in each round is illuminated as follows. Firstly, each node in the cluster selects a random number between 0 and 1 . If the number is below a certain threshold, the node becomes the cluster head candidate. Then, the final cluster heads are determined by using the competitive algorithm, and new cluster heads broadcast the messages to the surrounding nodes. Each node determines which cluster to join in based on the competitive function and replies to the messages to the cluster head.

In the data transmission phase, all nodes in the cluster send data to the cluster head in accordance with TDMA (Time Division Multiple Access) slots. Cluster head fuses the data and then transmits to the base station. After a period of continuous operation, the network reenters the start-ups phase and begins the next round of the cluster heads selection and reestablishes the clusters.

3.1. Reputation Evaluation. Due to the limited energy, some nodes are selfish in order to save their own energy. So it is important to consider the reputation paradigm for network nodes. The lower the reputation value is, the more selfish the nodes are. Therefore, the node's reputation value is one of the factors that need to be considered in the selection of cluster head.

Node reputation value could be computed by similarity of collected data and the historical interaction experience between nodes. Moreover, when the nodes compete to become the cluster head, they can charge a certain reputation value from other nodes as the compensation of energy consumption.

3.1.1. Calculation of Direct Reputation Value. Direct reputation is obtained by listening to the direct interaction of two nodes. It uses the historical interaction experience between nodes. The more successful interactions of node $i$ to node $j$ are, the higher reliability of node $i$ to node $j$ is. In a period of time, if the number of interactions from node $i$ to node $j$ is $N$ and the number of successful interactions is $k$, the reputation by direct interaction of node $i$ to node $j$ could be expressed as

$$
\varphi_{i j}= \begin{cases}0.5+\frac{k-(N-k)}{2 N_{0}}, & N<N_{0} \\ \frac{k}{N}, & N \geq N_{0},\end{cases}
$$

where $N_{0}$ is the threshold of interaction frequency.

Generally, interaction time is very important to the reliability of the evaluation and reputation value. Therefore, the interaction time of node $i$ and node $j$ is divided into $R$ segments. For the collected information in paragraph $r$ of node $i$, the time attenuation factor $\rho_{r}$ and the importance factor $V_{r}$ are introduced to calculate the direct reputation value. Direct reputation value of node $i$ could be expressed as

$$
\operatorname{DRV}_{i}=\frac{\sum_{j=1}^{M} \sum_{r=1}^{R} \varphi_{i j} \cdot \rho_{r} \cdot V_{r}}{R},
$$

where $M$ is the number of neighbor nodes of node $i$.

3.1.2. Calculation of Relative Reputation Value. In CWSNS, since the distribution of nodes is relatively dense, the observational data by the neighboring nodes often has a lot of similarities. Comparing the data of the neighbor nodes in the same period, we can see that the higher degree of similarity is, the more reliable it is.

Assume that the feature vector of environmental data collected by node $i$ in a period of time is expressed as $D_{i}=$ $(t, e, a, v)$, where $t$ represents the type of object (the value range is set as $T$ ), $e$ represents the event type (the value range is set as $E$ ), $a$ represents the attribute of the observation area (the value range is set as $A$ ), and $v$ represents the value of perceptual information (the value range is set as $V$ ).

The relative reputation between nodes is calculated by the similarity of data feature vectors. There are two adjacent nodes $i$ and $j$. Their data feature vectors are $\mathrm{DF}_{i}=\left(t_{i}, e_{i}, a_{i}, v_{i}\right)$ and $\mathrm{DF}_{j}=\left(t_{j}, e_{j}, a_{j}, v_{j}\right)$. The similarity of data feature vectors of node $i$ and node $j$ in a period of time is

$$
\operatorname{Sim}\left(\mathrm{DF}_{i}, \mathrm{DF}_{j}\right)=\cos \left(\overrightarrow{\mathrm{DF}}_{i}, \overrightarrow{\mathrm{DF}}_{j}\right)=\frac{\overrightarrow{\mathrm{DF}}_{i} \cdot \overrightarrow{\mathrm{DF}}_{j}}{\left|\overrightarrow{\mathrm{DF}}_{i}\right|\left|\overrightarrow{\mathrm{DF}}_{j}\right|}
$$

The relative reputation value of $i$ in this period of time is

$$
\mathrm{RRV}_{i}=\frac{\sum_{j=1}^{M} \lambda\left(1+\operatorname{Sim}\left(\mathrm{DF}_{i}, \mathrm{DF}_{j}\right)\right)}{M},
$$

where $M$ is the number of nodes adjacent to node $i$ and $\lambda>0$ is similar parameters to calculate relative reputation value. 
3.1.3. Calculation of Reputation Value. The reputation value of node $i$ is composed of direct reputation value (DRV), relative reputation value (RRV), and income and expenses value (IEV). It is expressed as

$$
\mathrm{RV}_{i}=\omega_{1} \mathrm{DRV}_{i}+\omega_{2} \mathrm{RRV}_{i}+\omega_{3} \mathrm{IEV}_{i},
$$

where $\omega_{1}+\omega_{2}+\omega_{3}=1$. If the node $i$ is a cluster head node, when member nodes want to join into the cluster, they need to pay some virtual reputation value to the cluster head node, where $\mathrm{IEV}_{i} \geq 0$; on the contrary, if node $i$ is a normal node and requests to join into the cluster, $\mathrm{IEV}_{i}<0$.

\subsection{Establishment of Cluster}

3.2.1. Competitive Radius of Cluster Head. The energy consumption of cluster head is different in even clustering multihop routing. The cluster head nodes near the base station take on more data forwarding tasks and consume more energy. In order to balance the energy consumption of cluster head nodes and prolong the network life, R-bUCRP uses uneven clustering in the stage of cluster establishment. The closer to the base station a cluster is, the smaller its size is. The communication radius of the cluster head node is related to the distance to the base station. Competitive radius of cluster head node is defined as

$$
R_{i}=\left(1-\mu \frac{d_{\max }-d\left(s_{i}, \mathrm{BS}\right)}{d_{\max }-d_{\min }}\right) R_{\max },
$$

where $R_{i}$ represents the competitive radius of cluster head node $i, R_{\max }$ represents the maximum competitive radius of system setting, and $\mu$ is a constant whose value is $[0,1]$.

3.2.2. Competitive Function of Cluster Head. In the cluster head selection process, this paper constructs a competitive function to avoid the excessive energy consumption or the selfish nodes elected as the cluster head. The competitive function is shown in

$$
W_{i}=\alpha \frac{E_{\mathrm{res}}}{\overline{E_{\mathrm{res}}}}+\beta \frac{\mathrm{RV}}{\overline{\mathrm{RV}}},
$$

where $W_{i}$ is the comprehensive weight value of energy and reputation and the larger the value is, the more competitive to be cluster head the node is, $E_{\text {res }}$ represents the current residual energy of node $i, \overline{E_{\text {res }}}$ represents the average residual energy of nodes in the network area, RV represents the current reputation value of node $i$, and $\overline{\mathrm{RV}}$ represents the average reputation value of nodes in the network area.

\subsubsection{Competitive Function of Joining into the Cluster. After} the cluster heads are determined, the cluster head nodes broadcast the Cluster_Msg packet and then wait for other nodes to join in. In this paper, when a node selects a cluster to join in, we consider not only the residual energy of cluster head, but also the distance to the cluster head and distance between cluster head and base station. The competitive function of node $i$ is given as

$$
F\left(i, \mathrm{CH}_{i}\right)=\frac{E_{\text {res }}}{d\left(i, \mathrm{CH}_{i}\right)^{2}+d\left(\mathrm{CH}_{i}, \mathrm{BS}\right)^{2}},
$$

where $\mathrm{CH}_{i}$ is the cluster head node, $E_{\text {res }}$ is the residual energy of the cluster head node, $d\left(i, \mathrm{CH}_{i}\right)$ is the distance between the nodes $i$ and the cluster head node $\mathrm{CH}_{i}$, and $d\left(\mathrm{CH}_{i}, \mathrm{BS}\right)$ is the distance between the cluster head $\mathrm{CH}_{i}$ and the base station.

The cluster establishment process is to achieve the purpose of load balancing. When a node wants to join into a cluster, it considers the energy residual of cluster head and transmission distance. From (10), it can be seen that there are three factors to determine the probability of joining into the cluster, that is, energy residual of cluster head, the distance between the cluster head and the normal node, and distance between the cluster head and the base station. In order to avoid the extreme situations of difficult choice (a node encounters a long distance with large energy or a short distance with low energy), especially in the later period of the network lifetime, the algorithm could give priority to the cluster head with the large signal strength when the energy of cluster head candidate is less than a certain threshold.

3.2.4. Establishment Process of Cluster. Network node cluster establishment process includes the cluster head election process and the process of normal nodes joining into the cluster. The corresponding pseudocode is described as in Algorithm 1.

\section{Simulation and Result Analysis}

4.1. Simulation Environment. In order to evaluate the performance of the proposed algorithms, we use MATLAB to simulate LEACH, EEUC, MADSEC, and our R-bUCRP under the same conditions and compare their performances. Simulation scene is set as follows: 1500 nodes are distributed in the network area of $400 \mathrm{~m} \times 400 \mathrm{~m}$, the coordinates of the base station are $(200,500) \mathrm{m}$, and each node moves towards a random direction at a speed uniformly distributed $[0,20 \mathrm{~m} / \mathrm{s}]$. We use energy model of literature [22] in the simulation. Detailed parameter settings are shown in Table 1.

4.2. Result Analysis. Since R-bUCRP introduces the concept of the reputation into the clustering process of CWSNS, reducing energy consumption of normal nodes when they communicate with the selfish nodes, at the same time, it balances the energy consumption of nodes in the network by using uneven clustering method. Therefore, it can be seen from Figure 4 that the first node death time and network death time of R-bUCRP are significantly later than those of the LEACH protocol, EEUC protocol, and MADSEC protocol.

Figure 5 compares the changes of network residual energy by using four protocols over time. Smaller slope shows a slower rate of energy consumption and indicates a longer survival time. As can be seen from Figure 5, the energy 


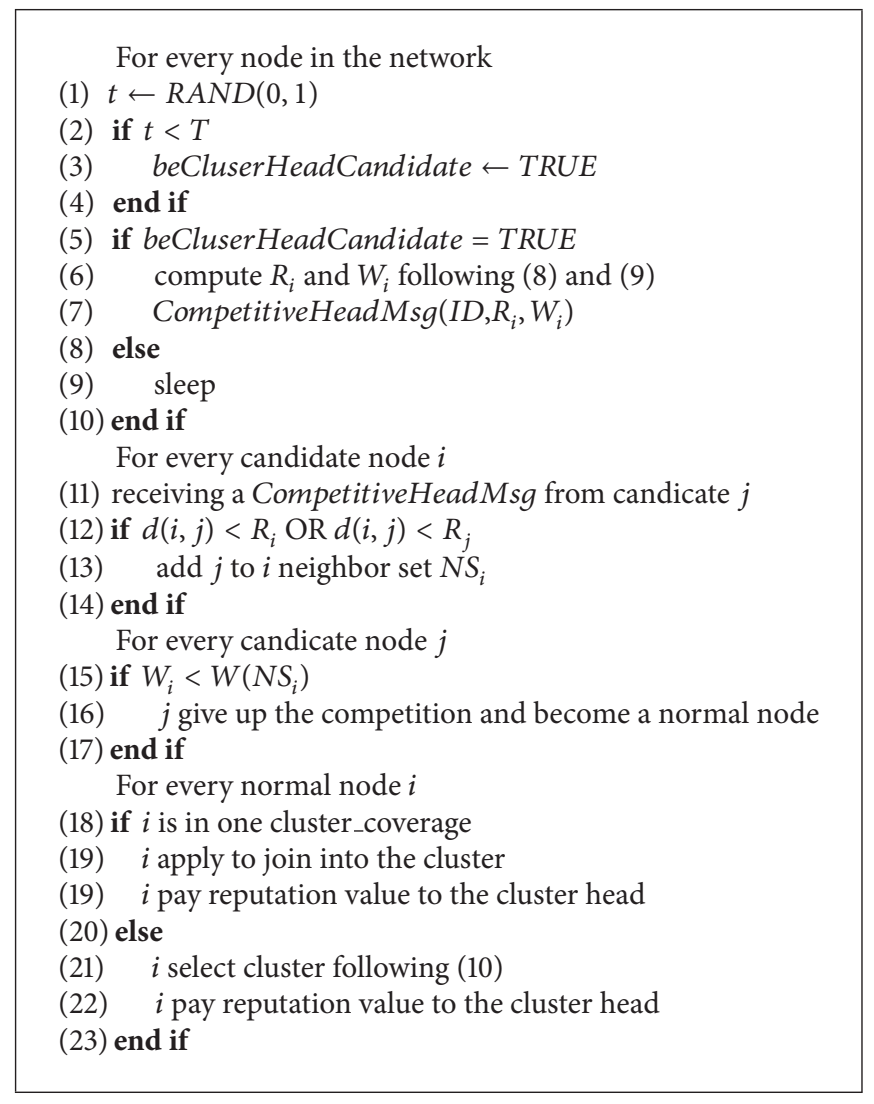

Algorithm 1: Cluster establishment.

TABLE 1: Network parameters and values.

\begin{tabular}{lc}
\hline Parameter & Value \\
\hline Network coverage & $(0,0) \sim(400,400) \mathrm{m}$ \\
Base station location & $(200,500) \mathrm{m}$ \\
Node number & 1500 \\
Initial energy & $0.5 \mathrm{~J}$ \\
Data packet size & $4000 \mathrm{bits}$ \\
Speed distribution & {$[0,20] \mathrm{m}$} \\
$E_{\text {elec }}$ & $50 \mathrm{~nJ} / \mathrm{bit}$ \\
$\varepsilon_{\mathrm{fs}}$ & $10 \mathrm{pJ} /\left(\mathrm{bit} \cdot \mathrm{m}^{2}\right)$ \\
$\varepsilon_{\mathrm{mp}}$ & $0.0013 \mathrm{pJ} /\left(\mathrm{bit} \cdot \mathrm{m}^{2}\right)$ \\
$E_{\mathrm{fusion}}$ & $5 \mathrm{~nJ} / \mathrm{bit}$ \\
\hline
\end{tabular}

consumption rate by using R-bUCRP is significantly smaller than the other three kinds of protocols, and the network has a longer survival time, with the better network quality.

Figure 6 shows the changes of node average energy by using four kinds of protocols over time. The node average energy by using R-bUCRP is higher than the other three kinds of protocols, which means R-bUCRP has a better performance in energy balance.

Figure 7 compares the changes of node average energy by using four kinds of protocols over the number of nodes in round 1000. With the increase of the node number in the network area, the node average energy is decreased.

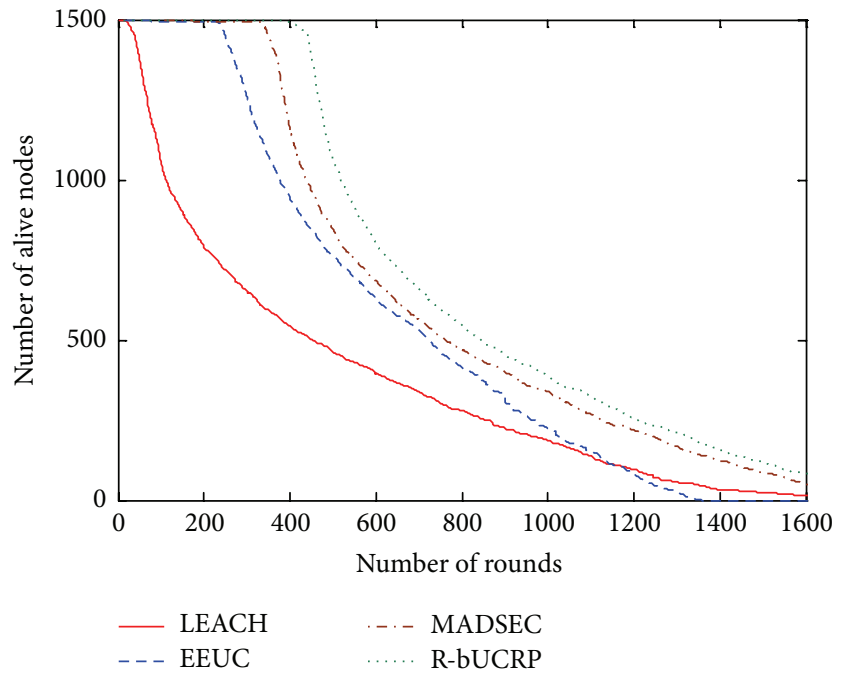

FIgURE 4: The relationship between the alive node number and round number.

As shown from Figure 7, the speed of energy consumption by using R-bUCRP is slower than the other three kinds of protocols, which means R-bUCRP has a better performance in scalability.

Figures 8, 9, 10, and 11 illustrate the stability of the algorithm. A stable clustering algorithm should also generate 


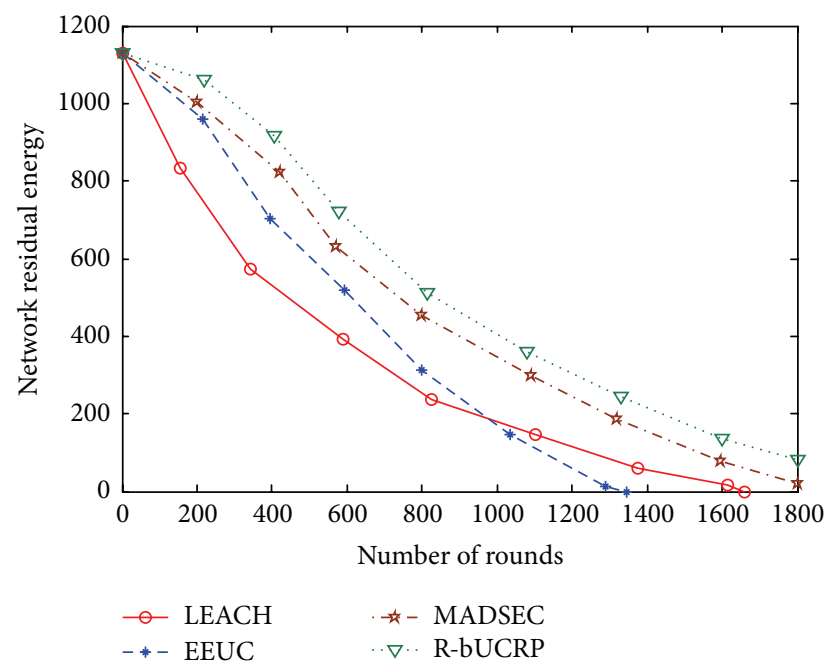

FIGURE 5: The relationship between network residual energy and round number.

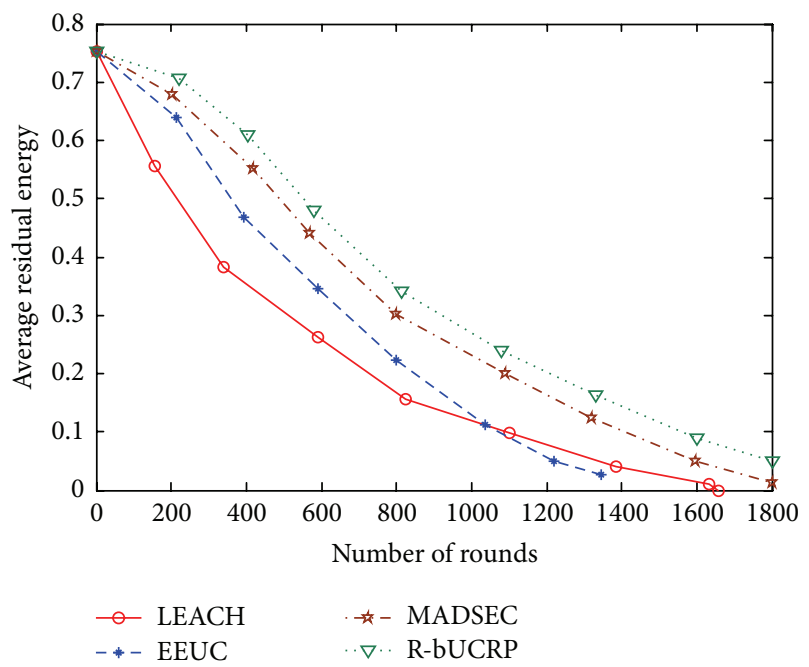

FIGURE 6: The relationship between average residual energy and round number.

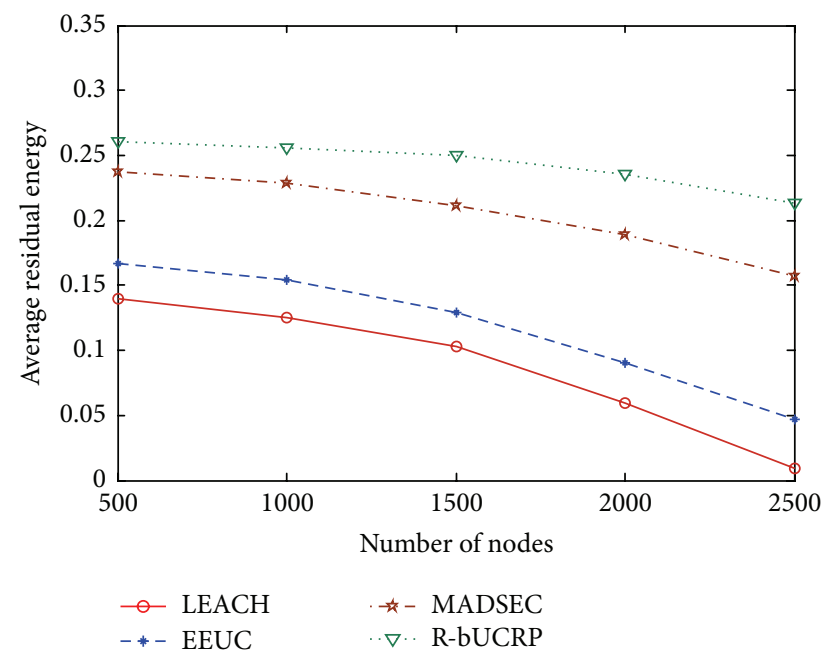

FIGURE 7: The relationship between average residual energy and node number.

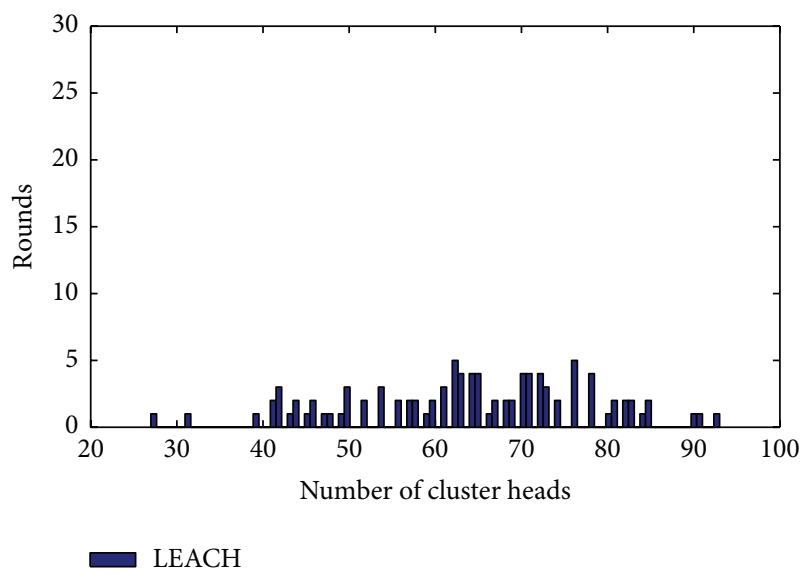

FIGURE 8: Cluster head number distribution of LEACH.

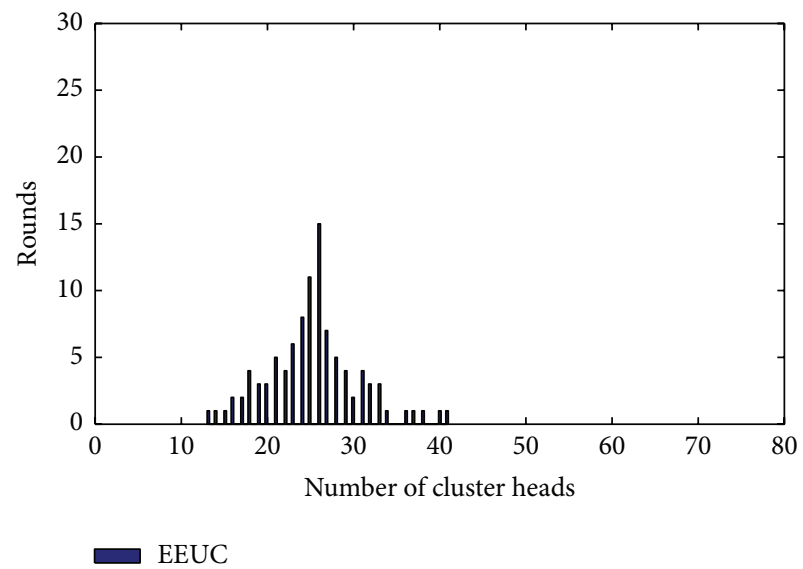

Figure 9: Cluster head number distribution of EEUC.

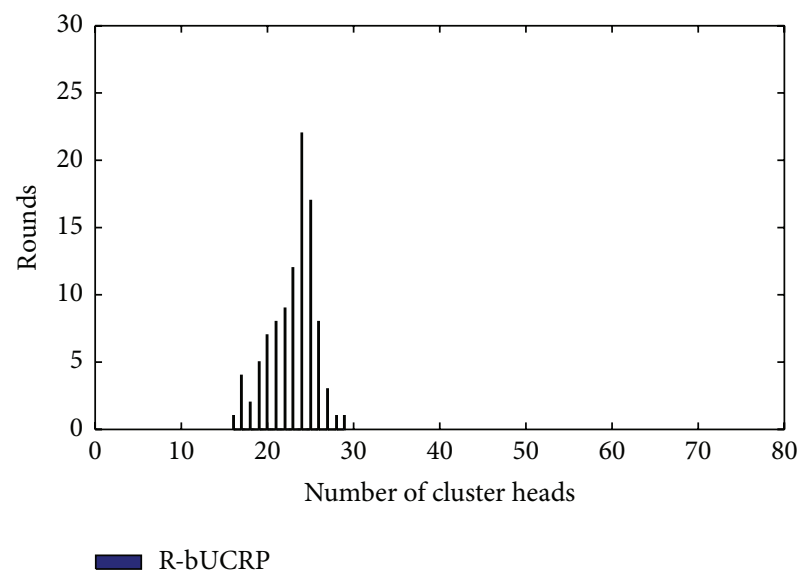

FIGURE 10: Cluster head number distribution of R-bUCRP.

more consistent number of clusters in order to balance the energy consumption of the network. Randomly select 100 rounds from the experimental processes of LEACH protocol, EEUC protocol, MADSEC protocol, and R-bUCRP, and count the distribution of cluster heads' number and the results are shown in Figures 8-11. Among these, LEACH 


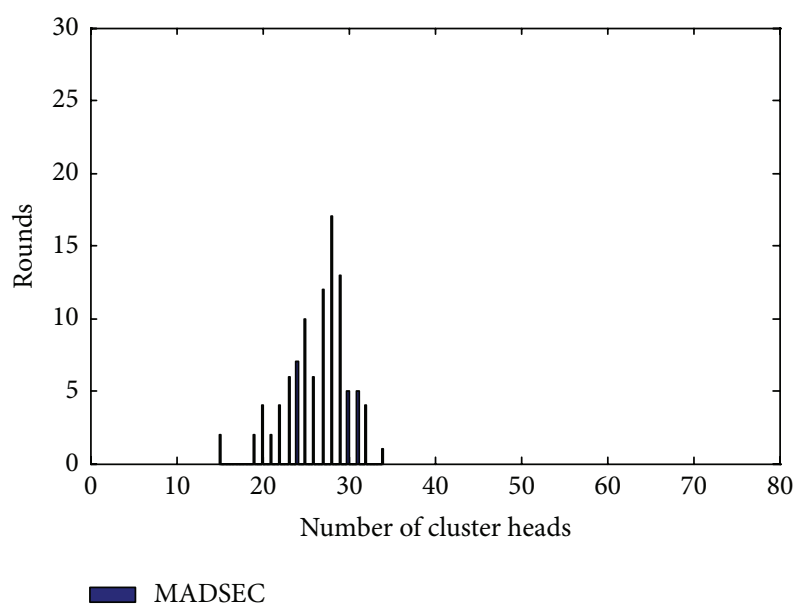

FIGURE 11: Cluster head number distribution of MADSEC.

protocol only uses the random number and threshold mechanism in the cluster head election; therefore, the range of cluster head number is large by using LEACH protocol. MADSEC protocol uses the $K$-means algorithm to divide the network area. EEUC protocol and R-bUCRP use the competitive function of cluster head candidates, and the number of cluster heads is controlled effectively within a certain range. In addition, R-bUCRP generates a more stable number of the cluster heads, which has better reliability.

\section{Conclusion}

A reputation-based uneven cluster routing protocol RbUCRP is proposed in this paper. The protocol adopts an uneven clustering method and introduces the reputation mechanism to reduce the communicational energy consumption between normal nodes and selfish nodes and increase the reliability of the network effectively. The algorithm optimizes the process of cluster head selection by competing for the cluster head among cluster head candidate nodes according to the residual energy and reputation value. Simulation results show that R-bUCRP can balance the network energy consumption, prolong the network lifetime, and increase the network reliability effectively.

\section{Competing Interests}

The authors declare that there are no competing interests regarding the publication of this paper.

\section{Acknowledgments}

This work is partially supported by the National Natural Science Foundation of China (NSFC) under Grants no. U1404611, no. U1204614, and no. 61370221, in part by the Program for Science \& Technology Innovation Talents in the University of Henan Province under Grants no. 16HASTIT035 and no. 14HASTIT045, in part by Program for Science \& Technology Innovative Research Team in University of Henan Province under Grant no. 14IRTSTHN021, and in part by Henan Science and Technology Innovation Outstanding Talent under Grant no. 164200510007.

\section{References}

[1] J. Mitola III and G. Q. Maguire Jr., "Cognitive radio: making software radios more personal," IEEE Personal Communications, vol. 6, no. 4, pp. 13-18, 1999.

[2] R. Urgaonkar and M. J. Neely, "Opportunistic scheduling with reliability guarantees in cognitive radio networks," in Proceedings of the IEEE Conference on Computer Communications (INFOCOM '08), Phoenix, Ariz, USA, April 2008.

[3] E. Dall'Anese, S.-J. Kim, G. B. Giannakis, and S. Pupolin, "Power allocation for cognitive radio networks under channel uncertainty," in Proceedings of the IEEE International Conference on Communications (ICC '11), pp. 1-6, Kyoto, Japan, June 2011.

[4] R. W. Thomas, D. H. Friend, L. A. DaSilva, and A. B. MacKenzie, "Cognitive networks: adaptation and learning to achieve endto-end performance objectives," IEEE Communications Magazine, vol. 44, no. 12, pp. 51-57, 2006.

[5] C. Fortuna and M. Mohorcic, "Trends in the development of communication networks: cognitive networks," Computer Networks, vol. 53, no. 9, pp. 1354-1376, 2009.

[6] A. Rabbachin, T. Q. S. Quek, H. Shin, and M. Z. Win, "Cognitive network interference," IEEE Journal on Selected Areas in Communications, vol. 29, no. 2, pp. 480-493, 2011.

[7] M. Zhang, H. Zhao, R. Zheng, Q. Wu, and W. Wei, "Cognitive internet of things: concepts and application example," International Journal of Computer Science Issues, vol. 9, no. 6, pp. 151158, 2012.

[8] M. Zhang, Y. Qiu, R. Zheng, X. Bai, W. Wei, and Q. Wu, "A novel architecture for cognitive internet of things," International Journal of Security and Its Applications, vol. 9, no. 9, pp. 235-252, 2015.

[9] R. Zheng, Q. Wu, M. Zhang, G. Li, J. Pu, and H. Wang, "A self optimization mechanism of system service performance based on autonomic computing," Computer Research and Development, vol. 48, no. 9, pp. 1676-1684, 2011 (Chinese).

[10] M. Zhang, R. Zheng, Q. Wu, W. Wei, X. Bai, and H. Zhao, "BiTRS: a bio-inspired trusted routing scheme for wireless sensor networks," Journal of Sensors, vol. 2015, Article ID 156843, 8 pages, 2015.

[11] R.-I. Ciobanu, C. Dobre, M. Dascălu, Ş. Trăuşan-Matu, and V. Cristea, "SENSE: a collaborative selfish node detection and incentive mechanism for opportunistic networks," Journal of Network and Computer Applications, vol. 41, no. 1, pp. 240-249, 2014.

[12] W. Guo, R.-Z. Xu, and B. Liu, "Research on subjective trust routing algorithm for mobile ad hoc networks," in Proceedings of the 6th International Conference on Wireless Communications, Networking and Mobile Computing (WiCOM '10), pp. 1-6, IEEE, Chengdu, China, September 2010.

[13] W. R. Heinzelman, A. Chandrakasan, and H. Balakrishnan, "Energy-efficient communication protocol for wireless microsensor networks," in Proceedings of the 33rd Annual Hawaii International Conference on System Siences (HICSS '33), Maui, Hawaii, USA, January 2000.

[14] A. Manjeshwar and D. Agrawal, "TEEN: a protocol for enhanced efficiency in wireless sensor networks," in Proceedings of the 1st International Workshop on Parallel and Distributed Computing Issues in Wireless Networks and Mobile Computing, San Francisco, Calif, USA, 2001. 
[15] A. Manjeshwar and D. P. Agarwal, "APTEEN: a hybrid protocol for efficient routing and comprehensive information retrieval in wireless sensor networks," in Proceedings of the International Parallel and Distributed Processing Symposium (IPDPS '02), pp. 195-202, Fort Lauderdale, Fla, USA, April 2002.

[16] L. Bhasker, "Genetically derived secure cluster-based data aggregation in wireless sensor networks," IET Information Security, vol. 8, no. 1, pp. 1-7, 2014.

[17] H. Lin and H. Uster, "Exact and heuristic algorithms for data-gathering cluster-based wireless sensor network design problem," IEEE/ACM Transactions on Networking, vol. 22, no. 3, pp. 903-916, 2014.

[18] P. T. A. Quang and D.-S. Kim, "An energy efficient clustering in heterogeneous wireless sensor and actuators networks," in Proceedings of the IEEE Globecom Workshops (GC Wkshps '12), pp. 524-528, Anaheim, Calif, USA, December 2012.

[19] K. Li and K. A. Hua, "Mobility-assisted distributed sensor clustering for energy-efficient wireless sensor networks," in Proceedings of the IEEE Global Communications Conference (GLOBECOM '13), pp. 316-321, IEEE, Atlanta, Ga, USA, December 2013.

[20] M. Ye, C. Li, G. Chen, and J. Wu, "EECS: an energy efficient clustering scheme in wireless sensor networks," in Proceedings of the 24th IEEE International Performance, Computing, and Communications Conference (PCCC '05), pp. 535-540, Phoenix, Ariz, USA, April 2005.

[21] S. Soro and W. Heinzelman, "Prolonging the lifetime of wireless sensor networks via unequal clustering," in Proceedings of the 5th IEEE International Workshop on Algorithms for Wireless, Mobile, Ad Hoc and Sensor Networks (WMAN '05), Denver, Colo, USA, 2005.

[22] C. F. Li, M. Ye, G. H. Chen, and J. Wu, "An energy-efficient unequal clustering mechanism for wireless sensor networks," in Proceedings of the 2nd IEEE International Conference on Mobile Ad-hoc and Sensor Systems (MASS '05), pp. 597-604, IEEE, Washington, DC, USA, November 2005.

[23] T. Chen, Y. Luo, F. Xiao, D. Shi, and S. Zhang, "Uneven clustering algorithm based on clustering optimization for wireless sensor networks," Computer Science, vol. 41, no. 6A, pp. 289292, 2014 (Chinese).

[24] C.-J. Jiang, W.-R. Shi, X.-L. Tang, P. Wang, and M. Xiang, "Energy-balanced unequal clustering routing protocol for wireless sensor networks," Journal of Software, vol. 23, no. 5, pp. 1222-1232, 2012 (Chinese).

[25] S. Buchegger and Y. J. Le Boudec, "The selfish node: increasing routing security for mobile ad hoc networks," LCA-Report 2001008, 2001.

[26] P. Naghizadeh and M. Liu, "Perceptions and truth: a mechanism design approach to crowd-sourcing reputation," IEEE/ACM Transactions on Networking, vol. 24, no. 1, pp. 163-175, 2016.

[27] Z. Li, H. Shen, and K. Sapra, "Leveraging social networks to combat collusion in reputation systems for peer-to-peer networks," IEEE Transactions on Computers, vol. 62, no. 9, pp. 1745-1759, 2013.

[28] M. T. Refaei, L. A. DaSilva, M. Eltoweissy, and T. Nadeem, "Adaptation of reputation management systems to dynamic network conditions in ad hoc networks," IEEE Transactions on Computers, vol. 59, no. 5, pp. 707-719, 2010.

[29] N. Labraoui, M. Gueroui, and L. Sekhri, "A risk-aware reputation-based trust management in wireless sensor networks," Wireless Personal Communications, vol. 87, no. 3, pp. 1037-1055, 2016.
[30] A. Capossele, G. De Cicco, and C. Petrioli, "R-CARP: a reputation based channel aware routing protocol for underwater acoustic sensor networks," in Proceedings of the International Conference on Underwater Networks \& Systems, Arlington, Va, USA, October 2015.

[31] P. Michiardi and R. Molva, "Core: a collaborative reputation mechanism to enforce node cooperation in mobile ad hoc networks," in Advanced Communications and Multimedia Security, B. Jerman-Blažič and T. Klobučar, Eds., vol. 100 of IFIP-The International Federation for Information Processing, pp. 107-121, Springer, New York, NY, USA, 2002.

[32] M. Zhang, C. Xu, J. Guan, R. Zheng, Q. Wu, and H. Zhang, "A novel Physarum-inspired routing protocol for wireless sensor networks," International Journal of Distributed Sensor Networks, vol. 2013, Article ID 483581, 12 pages, 2013.

[33] M. Zhang, C. Xu, J. Guan, R. Zheng, Q. Wu, and H. Zhang, "A novel bio-inspired trusted routing protocol for mobile wireless sensor networks," KSII Transactions on Internet and Information Systems, vol. 8, no. 1, pp. 74-90, 2014.

[34] Q. Wu, X. Zhang, M. Zhang, Y. Lou, R. Zheng, and W. Wei, "Reputation revision method for selecting cloud services based on prior knowledge and a market mechanism," The Scientific World Journal, vol. 2014, Article ID 617087, 9 pages, 2014. 


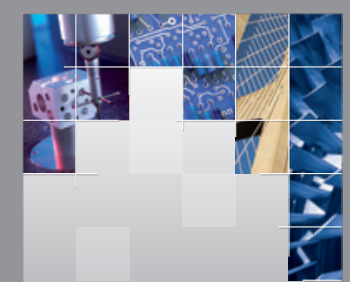

\section{Enfincering}
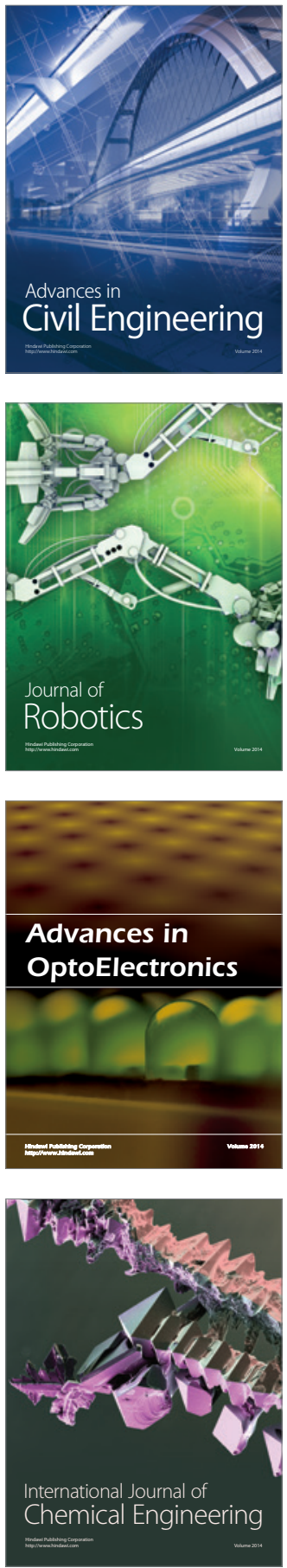

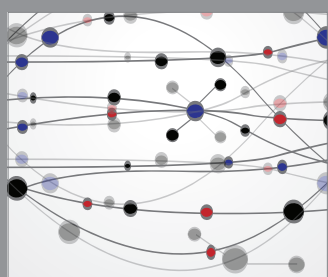

The Scientific World Journal

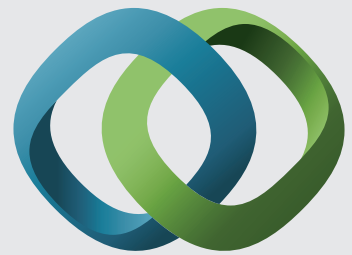

\section{Hindawi}

Submit your manuscripts at

http://www.hindawi.com
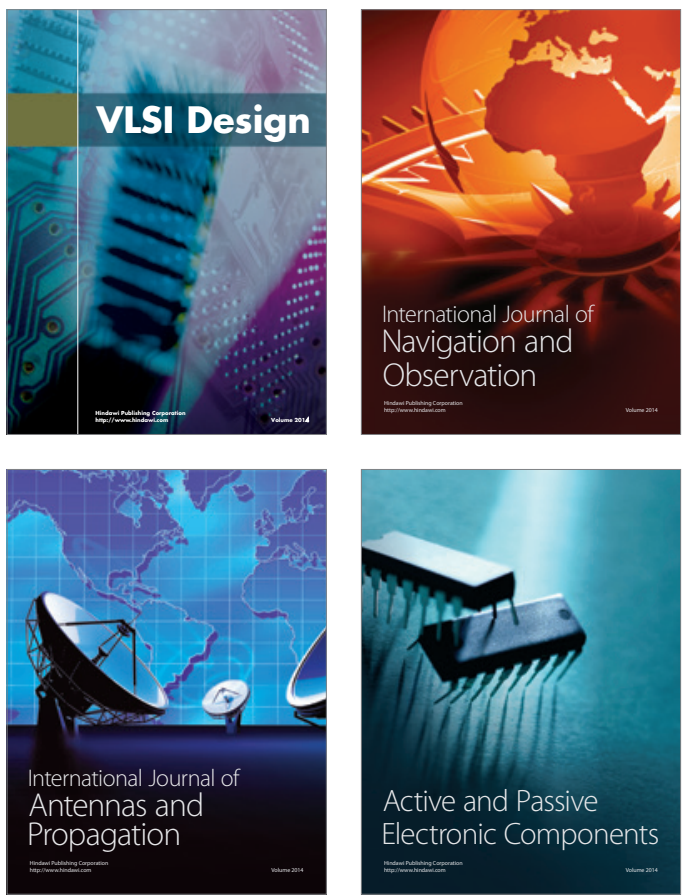
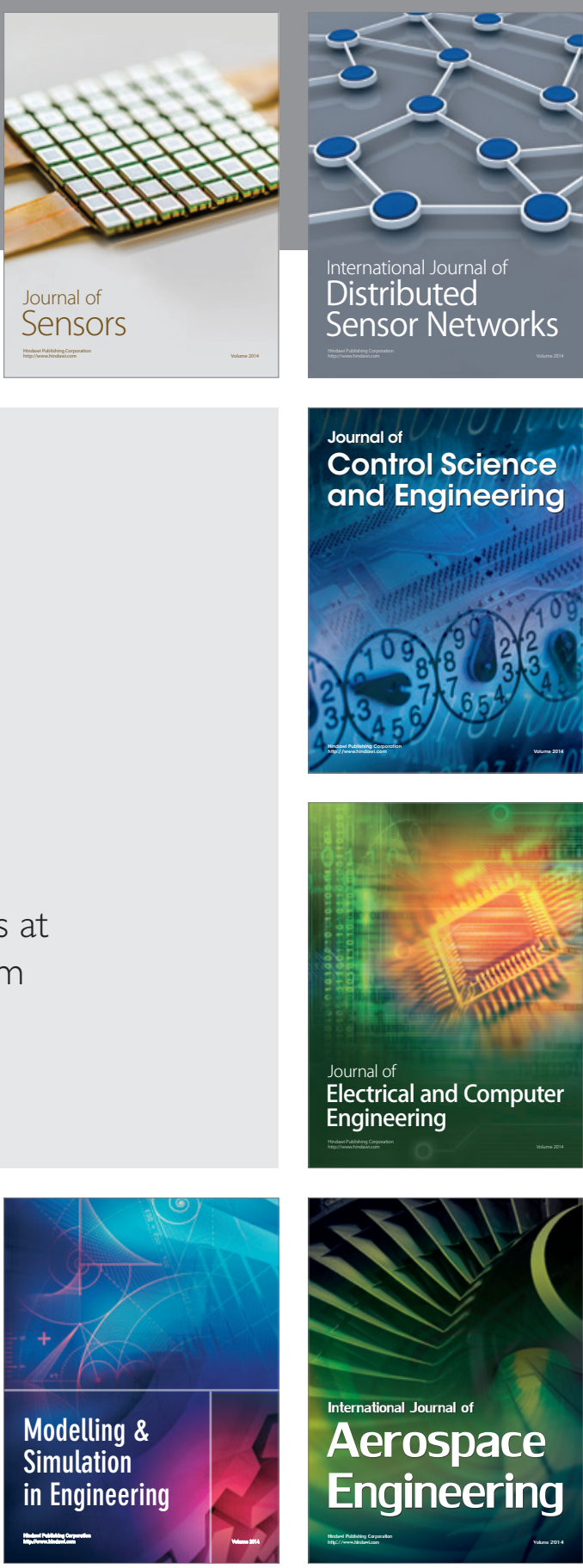

International Journal of

Distributed

Sensor Networks

Journal of

Control Science

and Engineering
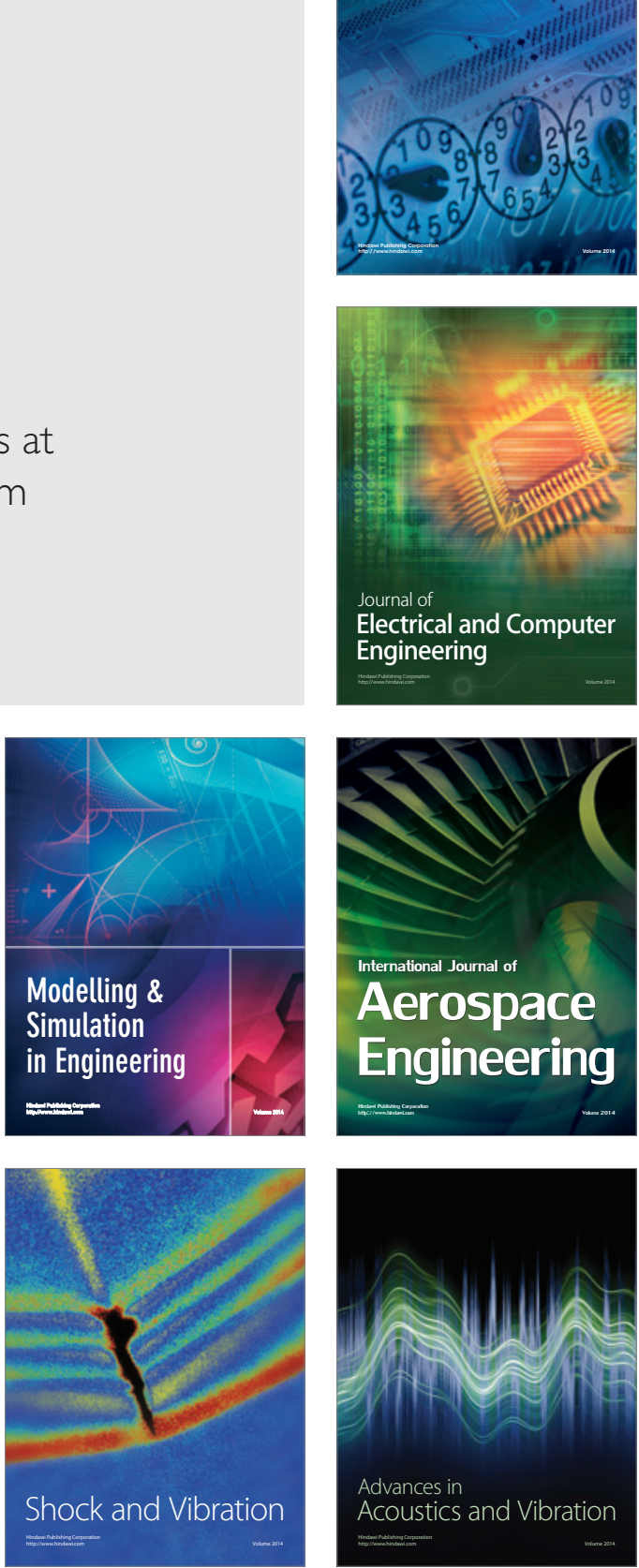